\title{
THE ROLE OF THE LEFT ATRIAL STRAIN PARAMETERS ON GRADING OF AORTIC REGURGITATION
}

\author{
Sedat Kalkan ${ }^{1}$, Suleyman Efe ${ }^{2}$, Onur TASAR ${ }^{3}$, Atilla Koyuncu ${ }^{4}$, Mehmet Yllmaz ${ }^{5}$, and \\ CAN KARABAY 6 \\ ${ }^{1}$ Pendik State Hospital \\ ${ }^{2}$ Kartal Kosuyolu Yuksek Ihtisas Heart-Education and Research Hospital \\ ${ }^{3}$ Saglik Bilimleri Universitesi Elazig Egitim ve Arastirma Hastanesi Rizaiye Binasi \\ ${ }^{4}$ Kartal Kosuyolu Heart and Research Hospital \\ ${ }^{5}$ Dr Siyami Ersek Thoracic and Cardiovascular Surgery Training and Research Hospital \\ ${ }^{6}$ KARTAL KOSUYOLU HEART AND RESEARCH HOSPITAL
}

May 21, 2020

\begin{abstract}
BACKGROUND:Aortic regurgitation(AR) is a clinical status characterized by diastolic blood backflow from aorta to the left ventricle. Grading the severity of AR is very important for clinical follow-up. In spite of numerous parameters, the severity of AR still remains unclear in some patients. In AR, left atrial(LA) mechanics can be affected in time and LA strain variations can be illuminating for the grading of AR. The purpose of this study is to determine whether the LA strain parameters are associated with the severity of aortic regurgitation or not. METHOD:64 consecutive patients with AR were included in this study.After echocardiographic examination according to the aortic regurgitation degree. Patients divided in three groups as mild(n: 22),moderate(n: 15) or severe(n: 27). All patients LA strain measurements performed and results compared between groups. RESULTS:According to echocardiographic measurements; between the groups; LA reservoir in the mild, moderate and severe AR groups were(42.0 $\pm 18.0,41.4 \pm 14.8,29.2 \pm 6.0$ repectively, p:0.002) and LA conduit in the mild, moderate and severe AR groups were $(21.2 \pm 8.7,19.3 \pm 7.4$ and $13.1 \pm 4.4$ respectively $\mathrm{p}<0.001)$, statistically significant different, while no statistically significant difference was noticed on LA SRs,LA SRe,LA SRa. CONCLUSION:This study showed that LA reservoir and LA conduit parameters of the patients with severe AR significantly decreased compared to those of the mild and moderate AR group. The grading of the LA mechanics in patients with chronic AR might provide a supplementary contribution to the present parameters in the grading of AR. Keywords: Aortic regurtation, graduation, left atrial strain
\end{abstract}

\section{INTRODUCTION}

Aortic regurgitation (AR) is a clinical status characterized by diastolic blood backflow from aorta to the left ventricle (LV) due to the anomalies of valve cusps and aortic root and/or aortic annulus. (1) Severe chronic AR, by causing volume and pressure overload on left ventricle, is likely to lead to left ventricular enlargement, a decrease in ejection fraction $(\mathrm{EF})$ and processes resulting in death in the long term.

Echocardiography is the most significant diagnostic test in the assessment of aortic regurgitation. Echocardiography allows for the examination of the anatomy of valve cusps and aortic root, the establishment of the diagnosis and severity of aortic regurgitation and the assessment of the LV diameters and functions. (1)

Grading the severity of AR is very important for clinical follow-up and determining an optimal surgical date. Clinical and echocardiographic parameters are used for the grading of AR. In spite of numerous parameters, the severity of AR still remains unclear in some patients. New parameters that will be proposed with current 
improvements and tools in echocardiographic assessment might provide a supplementary contribution to the grading of AR.

The significance of the left atrium (LA) in cardiovascular performance has been recognized for a long time. In recent years, new visualization techniques that present a supplementary view for the assessment of the left atrium have been introduced. Atrial strain and strain rate analysis, which are obtained by tissue Doppler imaging or two-dimensional speckle tracking echocardiography have been proved to be practical and repeatable for assessing the LA mechanics. (3) In AR, which causes severe volume and pressure overload on LV, LA mechanics can be affected in time in parallel with the elevated LV end-diastolic pressure (LVEDP), and LA strain variations can be illuminating for the grading of AR.

The purpose of this study is to determine whether the LA strain parameters are associated with the severity of aortic regurgitation or not.

\section{METHODOLOGY}

\section{Patient Population}

AR patients who applied to the echocardiography laboratory of our hospital within a year were examined. Following the ethics approval of the local ethics committee, the consent of the patients who were included in the study was obtained. 98 patients were examined in our study.

18 patients were excluded from the study due to the coexisting additional cardiac pathology (more than mild), 6 due to the low image quality and 10 due to the fact that the specialists who performed the examination had different opinions about the degree of regurgitation. 64 patients were included in our study after using exclusion criteria (Figure 1). Patients divided according to the aortic regurgitation degree in three groups as mild (n: 22) - moderate (n: 15) or severe (n: 27). All of the patients who met both exclusion and inclusion criteria were consecutively included in the study.

\section{FLOW CHART Figure 1}

\section{Exclusion criteria}

1. Patients with acute AR or decompensated valvular failure

2. Other concomitant valvular diseases of more than mild in severity,

3. Known ischemic heart disease,

4. Chronic pulmonary obstructive disease

5. Previous cardiac or valve surgery,

6. Reduced LVEF ([?]50\%), and

7. Inadequate echocardiographic data for 2D-STE analysis were excluded.

\section{Clinical characteristics}

All patients included in the study were subjected to a complete physical examination. Following the measurement of height and weight anthropometrically, body surface area $\left(\mathrm{BSA}, \mathrm{m}^{2}\right)$ and body mass index (BMI, $\mathrm{kg} / \mathrm{m}^{2}$ ) were calculated for each patient. After resting in a quiet room for $10 \mathrm{~min}$, the blood pressures of the patients were measured.

\section{Echocardiography}

Transthoracic echocardiographic images were obtained using an ultrasound system, Vivid-7 (General Electric Vingmed), from the patients in the left lateral decubitus position, and these images were digitally kept for offline examination (EchoPAC version 110.0.0, GE-Vingmed). Echocardiographic acquisitions (colored, standard 2D, pulsed, and continuous-wave Doppler) were done. Standard M-mode images at a parasternal long-axis view were used to obtain the LV dimensions. Then, LV end-diastolic and end-systolic volumes were calculated using biplane Simpson's method from the apical views (two- and four-chamber), and a correction was also performed for BSA. Following the aforementioned measurements, LVEF was calculated and the 
results were expressed with percentage. The biplane area-length method was used for the measurement of the LA volume (4).

All measurements and evaluations performed in the study were carried out considering the guidelines of the European Society of Echocardiography. Detailed examination of the aortic root, AV, and proximal ascending aorta was performed taking into account the standard guidelines. To evaluate the AR severity, comprehensive, color, continuous, and pulsed-wave Doppler recordings were carried out considering the recommendations that included the measurement of regurgitant jet width, vena contracta width, pressure half-time, and diastolic flow reversal in the descending aorta (5-7)

Aortic regurgitation severity was fixed on by two expert cardiologists who were working in the echocardiography laboratory with more than 5 years of experience. They used conventional echocardiographic evaluation methods for deciding severity degree if both of them were in the same decision on the severity degree of the patients in the study.

\section{Left Atrial Strain Parameters}

2D grayscale images of three cardiac cycles from apical four-chamber views were obtained with the help of conventional ultrasound, with a frame rate of 60-90 frames/s. The lateral and septal walls of the LA were continuously imaged without important dropouts. Two experienced cardiologists who were blinded to the study groups carried out the examinations. In order to calculate the strain rate and the LA strain, the off-line EchoPAc workstation was used by the researchers. As a reference point, the LA 2D-STE curves were acquired using the R-wave onset of the electrocardiogram. Following the manual definition of the endocardial border, the software automatically developed the tracing for each view. When the automatically acquired tracking segments were sufficient for the analysis, the researchers allowed the software to read the data. For insufficient tracking segments, manual adjusting or software parameters changing were performed.

As the average of six segmental values, strain rate parameters and global LA strain were evaluated. QRS onset speckle-tracking echocardiography analysis for LA; LA peak strain prior to mitral valve opening was taken as LA reservoir (LA-Res), and LA strain prior to atrial contraction (onset of the P-wave on electrocardiography) was taken as LA-conduit. LA strain rate throughout ventricular systole (LA-SRs), LA-SRe throughout ventricular passive filling and LA-SRa throughout active atrial contraction were measured as LA function parameters.

\section{RESULTS}

In our study, we researched the role of the LA strain parameters on the grading of AR. According to the grading, the patients were divided into three groups: mild, moderate, and severe AR. The basal variables among these groups are presented in Table 1. Except for gender, no statistically significant difference was noticed on other parameters among the groups.

Echocardiographic parameters are defined in Table 2. According to these parameters, end-diastolic volume (EDV) was detected to be $103.7 \pm 35.7 \mathrm{~mL}$ in the mild AR group, $119.0 \pm 40.2 \mathrm{~mL}$ in the moderate AR group and $167.0 \pm 59.7 \mathrm{~mL}$ in the severe AR group $(\mathrm{p}<0.001)$. End-systolic volume $(\mathrm{ESV})$ was detected to be $39.1 \pm 16.6$ in the mild AR group, $46 \pm 20.4 \mathrm{~mL}$ in the moderate AR group and $65.9 \pm 27.4 \mathrm{~mL}$ in the severe AR group $(\mathrm{p}<0.001)$. AR vena contracta $(\mathrm{VC})$ was detected to be $0.3 \pm 0.1 \mathrm{~cm}$ in the mild AR group, $0.4 \pm 0.1 \mathrm{~cm}$ in the moderate AR group and $0.7 \pm 0.1 \mathrm{~cm}(\mathrm{p}$ value $<0.001)$ in the severe AR group. Left atrium volume (LAV) was detected to be $46.7 \pm 9.1 \mathrm{~mL}$ in the mild AR group, $46.5 \pm 10.1 \mathrm{~mL}$ in the moderate AR group and $59.5 \pm 10.7 \mathrm{~mL}$ in the severe AR group with a p value of 0.005 .

In Table 3, left atrial mechanics are listed, and, between the groups; reservoir (42.0 \pm 18.0 in the mild AR group, $41.4 \pm 14.8$ in the moderate AR group and $29.2 \pm 6.0$ in the severe AR group with a palue of 0.002$)$ and conduit ( $21.2 \pm 8.7$ in the mild AR group, 19.3 \pm 7.4 in the moderate AR group and $13.1 \pm 4.4$ in the severe AR group ( $\mathrm{p}$ value $<0.001)$ ), statistically significant difference was noticed, while no statistically significant difference was noticed on LA SRs, LASRe, LA SRa. 
With the post-hoc Tukey analysis on the grading of AR, the $\mathrm{p}$ value of mild and moderate AR for the LA reservoir was determined to be 0,98 , while the $\mathrm{p}$ value of mild and severe AR was determined to be 0,004 and the $\mathrm{p}$ value of moderate and severe AR was determined to be 0,02 . The $\mathrm{p}$ value of mild and moderate AR for LA conduit was determined to be 0,69 , while mild to severe AR was determined to be $\mathrm{p}<0,001$ and the $\mathrm{p}$ value of moderate to severe AR was determined to be 0,02 .

For EDV, the $\mathrm{p}$ value of mild to moderate AR was determined to be 0,62 , whereas mild to severe AR was determined to be $\mathrm{p}<0,001$ and the $\mathrm{p}$ value of moderate to severe AR was determined to be $\mathrm{p} 0,01$. For ESV, the $\mathrm{p}$ value of mild to moderate AR was determined to be 0,58 , whereas mild to severe AR was determined to be $p<0,001$ and the $p$ value of moderate to severe AR was determined to be 0,03 . For AR VC, the p value of mild to moderate AR was determined to be 0,01 , while mild to severe AR was determined to be $\mathrm{p}<0,001$ and moderate to severe AR was determined to be $\mathrm{p}<0,001$. For LAV, the p value of mild to moderate AR was determined to be 0,99 , while the $\mathrm{p}$ value of mild to severe AR was determined to be $\mathrm{p} 0,01$ and moderate to severe AR was determined to be 0,02 .

\section{DISCUSSION}

In our study, basically; the facts that

1. LA mechanics were disrupted in AR patients

2. LA reservoir and LA conduit decreased as the severity of AR increased

3. LA reservoir and LA conduit significantly decreased in severe AR compared to mild and moderate AR were determined.

The grading of AR for the patient's follow-up and planning the treatment after the diagnosis of AR is critical. Patients with normal levels of LV diameters and functions, and who are asymptomatic with mild and moderate AR do not need to be treated, and a 12-to-24-month echocardiographic follow-up period is suggested for them. On the other hand, patients with normal LV functions, and who are asymptomatic with severe aortic regurgitation are supposed to be examined at a 6-month period. (8)

The grading of AR needs to be done with many clinical and echocardiographic parameters, without separating them. Some of the echocardiographic parameters are EROA, regurgitant volume, Jet/LVOT ratio, VC, holodiastolic flow reversal, LV dilatation, and AR PHT. Despite all of these parameters that are in use for the grading of AR, an ultimate decision cannot be taken in some cases and the severity of AR cannot be clarified. Each unit decrease in LA strain value was demonstrated to increase the likelihood of the progress of pulmonary HT in AR patients by 6\%. (9) These data show the importance of the LA mechanics in chronic AR. In our study, it was noticed that LA reservoir and LA conduit significantly decreased in severe AR patients compared to the patients with mild and moderate AR, and that LA reservoir and LA conduit might contribute to the grading of AR.

Some of the parameters used for the grading of AR, and, especially AR PHT value were associated with elevated LVEDP, and they were inversely correlated. In other words, as LVEDP increases, AR PHT value decreases, and the values below $200 \mathrm{~ms}$ are interpreted to be related to severe AR. Elevated LVEDP present in AR is closely related to the LA mechanics, and decreased LA strain is an independent predictor of elevated LVEDP. (10) LA dimension might be considered to be an approximate indicator of LV diastolic filling pressures. (11)

In healthy individuals, the left atrium is quite flexible in exposure to rather low pressures; however, it becomes tense and stiff in case of acute and chronic damage. $(12,13,14)$. The left atrium is directly exposed to LV vacuum pressure during diastole; therefore, in the absence of LA volume overload, an enlarged left atrium is a strong indicator of elevated LV filling pressure, which also explains the causality between LA dilatation and negative results. (11)

LA reservoir is significantly associated with systolic performance measurements such as ejection fraction and LV systolic volume index. In other words, both diastolic (LVEDP) and systolic (LV systolic volume index) LV variables are independent predictors of the LA reservoir. (15) Besides, the LA reservoir presents more 
precise information than the LA volume index and other Doppler dependent variables for the assessment of LVEDP.

Severe chronic AR causes pressure and volume overload on the left ventricle. According to LaPlace's law, wall stress is about the division of wall thickness by intraventricular pressure and the radius. LV dilatation increases LV systolic wall stress, which is required for obtaining the systolic pressure level similar to the one obtained at normal ventricular diameter. Therefore, in chronic AR, both preload and afterload increase simultaneously. LV systolic function is preserved with LV dilatation and hypertrophy. The amount of regurgitant volume is directly associated with volume overload, and it is directly proportional with the severity of the leak. While mild AR causes volume overload minimally, severe AR might result in progressive circles dilatation due to massive volume overload. (1)

Extreme volume overload in AR causes the dysfunction of the myocardial mechanics insidiously. (16) In compensated severe AR, extreme volume overload is adjusted by eccentric hypertrophy, which includes lengthened myofibrils. $(17,18)$ In spite of elevated regurgitant volume, diastolic adaptation is preserved by keeping the LV filling pressures at normal or mildly-elevated levels with eccentric hypertrophy. Meanwhile, $\mathrm{EF}$ is maintained at normal levels by balancing increased volume overload with increased LV bulk. (19) Even though it was compensated, in mild and moderate AR, it was demonstrated that global LV performance significantly decreased despite normal EF values. (20) Thus, in order an optimal surgical date to be determined, new indicators that would show subclinical dysfunction are required. (16)

In decompensated AR, decompensation progresses due to increased interstitial fibrosis and decreased compliance, and pressure and volume increase after LV systole. LV diastolic compliance decreases due to hypertrophy and fibrosis, and it coexists with systolic function. This condition causes high filling pressures and symptoms of heart failure.

At the stages of decompensation; left atrial, pulmonary capillary wedge pressure, the pressures in the right heart and pulmonary artery increase, and cardiac output begins to decrease firstly during exercise, then during rest. Increased LA pressure is directly associated with regurgitant volume, and it can be stated that this association is continuous. Hence, in parallel with the facts we obtained with the LA mechanics, it can be suggested that LA strain parameters might contribute to the grading of AR.

When the pathophysiology of chronic AR is considered, it is not surprising that elevated LVEDP and LA strain parameters, which increase with the severity of AR, are affected. In our study, it was demonstrated that among the patients whose diseases were classified as mild, moderate, and severe AR, LA reservoir and LA conduit values of the severe AR group significantly decreased compared to those of the mild and moderate group. The decrease was not significant for the difference between the mild and moderate. It is possible that LV compensatory capacity is not sufficient due to severe LV overload, and as LVEDP increases, it causes the LA mechanics and strain parameters to be affected in time. When considered from this point of view, LA reservoir and LA conduit parameters can provide a supplementary contribution to and be illuminating for EKO parameters, which have been clinical and in use for the grading of AR for a long time.

The fact that our study was monocentric and observational, that the number of the patients was not sufficient and the fact that LVEDP could not be calculated with invasive methods are some of the limitations of our study. However, the fact that a group of patients whom could only be rarely encountered in clinical practice like isolated AY were chosen, the likelihood that a routinely-performed invasive procedure would increase the complication risk in the present group of patients and some ethical issues led to these limitations. Polycentric studies that can be carried out with a larger group of patients can help us to obtain more precise facts on this issue.

\section{CONCLUSION}

In our study, it was detected that the LA reservoir and LA conduit parameters of the patients with severe AR significantly decreased compared to those of the mild and moderate AR group. The grading of the LA mechanics in patients with chronic AR might provide a supplementary contribution to the present parameters 
with a holistic approach in the grading of AR.

\section{REFERENCE}

1.Bekeredjian R, Grayburn PA. Valvular heart disease: aortic regurgitation. Circulation. 2005 Jul 5;112(1):125-34. Review. Erratum in: Circulation. 2005 Aug 30;112(9):e124. PubMed PMID: 15998697.

2.Cohn LH, Birjiniuk V. Therapy of acute aortic regurgitation. Cardiol Clin . 1991;9:339-352.

3.Vieira MJ, Teixeira R, Gonçalves L, Gersh BJ. Left atrial mechanics:echocardiographic assessment and clinical implications. J Am Soc Echocardiogr. 2014 May;27(5):463-78. doi: 10.1016/j.echo.2014.01.021. Epub 2014 Mar 20. Review. PubMed PMID: 24656882.

4.Pritchett, A. M., Jacobsen, S. J., Mahoney, D. W., Rodeheffer, R. J., Bailey, K. R., \& Redfield, M. M. (2003). Left atrial volume as an index of left atrial size: A population-based study. Journal of the American College of Cardiology, 41, 1036-1043.

5.Lancellotti P Tribouilloy C Hagendorff A Moura L Popescu BA Agricola Eet al. European Association of Echocardiography recommendations for the assessment of valvular regurgitation. Part 1: aortic and pulmonary regurgitation (native valve disease). Eur J Echocardiogr 2010;11:223-44

6.Zoghbi WA Enriquez-Sarano M Foster E Grayburn PA Kraft CD Levine RAet al. Recommendations for evaluation of the severity of native valvular regurgitation with two-dimensional and Doppler echocardiography. J Am Soc Echocardiogr 2003;16:777-802

7.Lang RM Bierig M Devereux RB Flachskampf FA Foster E Pellikka PAet al. Recommendations for chamber quantification. Eur J Echocardiogr 2006;7:79-108.

8.Aortic Valve Disease Brian R. Lindman, Robert O. Bonow and Catherine M.Otto

9.Salas-Pacheco JL, Avila-Vanzzini N, Eugenia RM, Arias-Godinez JA. Left atrium function by 2D speckle tracking in aortic valve disease.Echocardiography . 2016;33(12):1828-1834.

10.Kurt M, Tanboga IH, Aksakal E, Kaya A, Isik T, Ekinci M, Bilen E. Relation of left ventricular enddiastolic pressure and N-terminal pro-brain natriuretic peptide level with left atrial deformation parameters. Eur Heart J Cardiovasc Imaging. 2012 Jun;13(6):524-30. doi: 10.1093/ejechocard/jer283. Epub 2011 Dec 13.PubMed PMID: 22166592.

11.Moller JE, Hillis GS, Oh JK, Seward JB, Reeder GS, Wright RS, et al. Left atrial volume: A powerful predictor of survival after acute myocardial infarction. Circulation 2003;107:2207-12.

12.Casaclang-Verzosa G, Gersh BJ, Tsang TS. Structural and functional remod- eling of the left atrium: Clinical and therapeutic implications for atrial fibrillation. J Am Coll Cardiol 2008;51:1-11.

13. Hoit BD, Shao Y, Gabel M. Left atrial systolic and diastolic function accom- panying chronic rapid pacing-induced atrial failure. Am J Physiol Heart Circ Physiol 1998;275:H183-9.

14. KhanA, MoeGW, NiliN, RezaeiE, EskandarianM, ButanyJ, etal.The cardiac atria are chambers of active remodeling and dynamic collagen turn-over during evolving heart failure. J Am Coll Cardiol 2004;43:68-76.

15.Wakami K, Ohte N, Asada K, Fukuta H, Goto T, Mukai S, et al. Correlation between left ventricular end-diastolic pressure and peak left atrial wall strain during left ventricular systole. J Am Soc Echocardiogr 2009;22: 847-51.

16. Cameli M, Sciaccaluga C, Mandoli GE, D'Ascenzi F, Tsioulpas C, Mondillo S. The role of the left atrial function in the surgical management of aortic and mitral valve disease. Echocardiography. 2019 Aug;36(8):1559-1565. doi: 10.1111/echo.14426. Epub 2019 Jul 1. Review. PubMed PMID: 31260141.

17.Ricci DR. Afterload mismatch and preload reserve in chronic aortic regurgitation. Circulation . 1982;66:826-834. 
18.Ross J Jr, McCullagh WH. Nature of enhanced performance of the dilated left ventricle during chronic volume overloading. Circ Res . 1972;30: 549-556.

19.Starling MR, Kirsh MM, Montgomery DG, Gross MD. Mechanism for left ventricular systolic dysfunction in aortic regurgitation: importance for predicting the functional response to aortic valve replacement. J Am Coll Cardiol. 1991; 17 : 887-897.

20. Sokmen G, Sokmen A, Duzenli A, Soylu A, Ozdemir K. Assessment of myocardialvelocities and global function of the left ventricle in asymptomatic patients with moderate-to-severe chronic aortic regurgitation: a tissue Doppler echocardiographic study. Echocardiography. 2007 Jul;24(6):609-14. PubMed PMID:17584200.

\section{Hosted file}

Table1.docx available at https://authorea.com/users/324960/articles/453013-the-role-of-theleft-atrial-strain-parameters-on-grading-of-aortic-regurgitation

\section{Hosted file}

Table 2.docx available at https://authorea.com/users/324960/articles/453013-the-role-of-theleft-atrial-strain-parameters-on-grading-of-aortic-regurgitation

\section{Hosted file}

Table 3.docx available at https://authorea.com/users/324960/articles/453013-the-role-of-theleft-atrial-strain-parameters-on-grading-of-aortic-regurgitation

\section{Hosted file}

Bel13.docx available at https://authorea.com/users/324960/articles/453013-the-role-of-theleft-atrial-strain-parameters-on-grading-of-aortic-regurgitation 